\title{
Analysis of Temperature Dependence of Thermally Induced Transient Effect in Interferometric Fiber-optic Gyroscopes
}

\author{
Woo-Seok Choi* \\ The 3rd R\&D Institute, Agency for Defense Development, Yuseong P.O. Box 35-3, \\ Daejeon 305-600, Korea
}

(Received June 15, 2011 : revised August 10, 2011 : accepted August 17, 2011)

\begin{abstract}
Thermal characteristics, such as diffusivity and temperature induced change in the fiber mode index of rotation sensing fiber coil are critical factors which determine the time varying, thermo-optically induced bias drift of interferometric fiber-optic gyroscopes (IFOGs). In this study, temperature dependence of the transient effect is analyzed in terms of the thermal characteristics of the fiber coil at three different temperatures. By applying an analytic model to the measured bias in the experiments, comprehensive thermal factors of the fiber coil could be extracted effectively. The validity of the model was confirmed by the fact that the extracted values are reasonable results in comparison with well known properties of the materials of the fiber coil. Temperature induced changes in the critical factors were confirmed to be essential in compensating the transient effect over a wide temperature range.
\end{abstract}

Keywords : Fiber-optic gyroscope, Shupe effect, Thermal characteristics of fiber coil OCIS codes : (060.2800) Gyroscopes; (060.2370) Fiber optic sensors; (120.5790) Sagnac effect; (120.6810) Thermal effect

\section{INTRODUCTION}

The interferometric fiber-optic gyroscope (IFOG) is a representative fiber-optic sensor based on the Sagnac effect to measure absolute rotation rates in inertial reference frames [1-2]. IFOGs have many advantages over conventional gyroscopes, such as long life, high reliability, light weight, low cost, and so on. Many useful research results related with IFOGs have been achieved so that the IFOG is now a good prospective candidate for future inertial navigation systems. However, in spite of such positive advances, there are still some difficulties to be overcome to improve IFOG performance.

The thermally induced non-reciprocity of IFOGs has been one of main obstacles to applying them in practical applications. This phenomenon, the so called Shupe effect, yields transient bias drift when a temporarily changing thermal gradient passes through the rotation-sensing fiber coil [3]. With the aid of invaluable earlier works, it could be proved that thermal transient non-reciprocity can be considerably reduced by applying a quadrupolar winding pattern to the fiber coil [4-5]. Nonetheless, this does not sufficiently ad- dress the problem because there can still be residual drift much larger than steady-state bias stability depending on the degree of imperfection in the thermal design and fabrication of the fiber coil. Quantitative analysis based on a thermal model considering heat conduction and physical stress is essential to identify proper methodologies which can address the imperfection [5-6]. Because thermal characteristics, such as diffusivity and temperature induced change in the effective index of the fiber coil, are critical factors determining the Shupe effect, it is necessary to examine the behavior of the thermal characteristics in detail to further improve the temporal thermal performance. However, all the established studies in analytic modeling of the Shupe effect have been performed with an assumption that thermal characteristics are invariant over temperature changes even though this is not true in the real world. For an accurate analysis of the thermal transient over a wide temperature range, it is indispensible to examine changes in thermal characteristics as a function of the average temperature measured during experiments.

In this study, temperature dependencies of the transient responses are analyzed focusing on changes in the thermal

\footnotetext{
*Corresponding author: cws@add.re.kr

Color versions of one or more of the figures in this paper are available online.
} 
characteristics of the fiber coil at several fiber coil temperatures. The related theory with analytic model is presented in Section 2, experimental methods, analyzed results, and a summary are given in Sections 3, 4, and 5, respectively.

\section{THEORY OF THE SHUPE EFFECT WITH ANALYTIC MODEL}

Non-reciprocity due to the transient temperature perturbation caused by differences between the coil temperature and ambient temperature, $\Delta T$, yields phase error expressed as $[5-6]$

$$
\Delta \phi_{e}=A \int_{0}^{L / 2}[\Delta \dot{T}(l)-\Delta \dot{T}(L-l)](2 l-L) d l
$$

where $A$ is the amplitude of the Shupe effect, $\Delta \dot{T}$ is the time derivative of $\Delta T, L$ and $l$ are the length of the fiber and the length of the curvilinear abscissa along the fiber, respectively. Eq. 1 is obvious under the assumption that the thermally induced temporal phase error is linearly proportional to the accumulated amount of non-uniform temperature change difference along the whole fiber. The amplitude, $A$ has the important role of describing phase imbalance between two counter-propagating optical beams along the Sagnac loop in terms of effective index and length variation of the fiber by thermal perturbations that can be expressed as

$$
A=\frac{k n}{c}\left(\frac{d n}{d T}+\alpha n\right) \approx \frac{k n}{c} \cdot \frac{d n}{d T}
$$

where $k$ is the wave vector in vacuum, $n$ is the mode index of the fiber, $\mathrm{c}$ is the speed of light in vacuum, and $\alpha$ is the linear thermal expansion coefficient of the fiber, respectively. Generally, as $\alpha$ is much smaller than one order of magnitude, the $\alpha n$ term is ignored in the following [7]. The critical factor, $d n / d T$, is not easy to know accurately in this model even though there is well-known information for the silica fiber itself. This is because the fiber experiences more severe physical circumstance when it is utilized in the fabrication of a fiber coil. As seen in Fig. 1, the fiber coil is usually comprised of various materials. Long coated fiber needs to be wound in a metallic spool and to be encapsulated by an adhesive potting material to make it a suitable coil with a homogeneous solid state. When some transient thermal perturbations are applied to the fiber coil, secondary effects such as stress or strain can occur due to elastic deformation caused by complex thermal interaction between the constituting materials [8-9]. The effective optical path along the fiber is also susceptible to such physical effects. Therefore, in this study, $d n / d T$ is not a fixed constant, but it is considered a critical factor that can change according to environmental conditions and effects resulting from the fabrication of the fiber coil.

For the thermal perturbation applied to the radial direction of the fiber coil with quadrupolar winding pattern, Eq. (1) can be rewritten as a discrete form:

$$
\begin{aligned}
& \Delta \phi_{e} / A=\sum_{i=1}^{N / 2}\left\{(-1)^{i+1}[\Delta \dot{T}(2 i-1)-\Delta \dot{T}(2 i)] .\right. \\
& \left.\left[\left(l_{i+(N / 2)-1}-l_{i+(N / 2)-2}\right)^{2}-\left(l_{i+(N / 2)-1}-l_{i+(N / 2)-2}\right) L\right]\right\}
\end{aligned}
$$

where $N$ is the total layer number, $\Delta \dot{T}(n)$ and $l_{n}$ are temperature change and the length in the $n$th fiber layer, respectively. To calculate the amount of accumulated transit phase error in Eq. (3), it is necessary to determine the $\Delta \dot{T}$

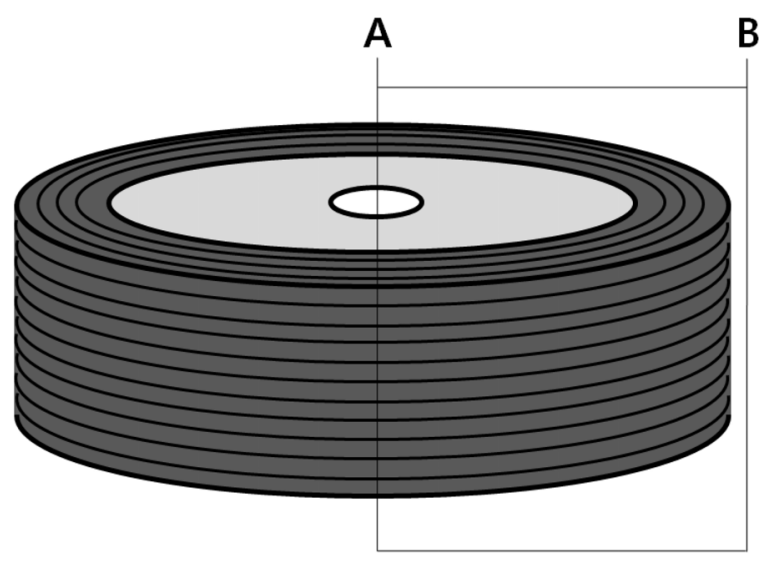

(a)

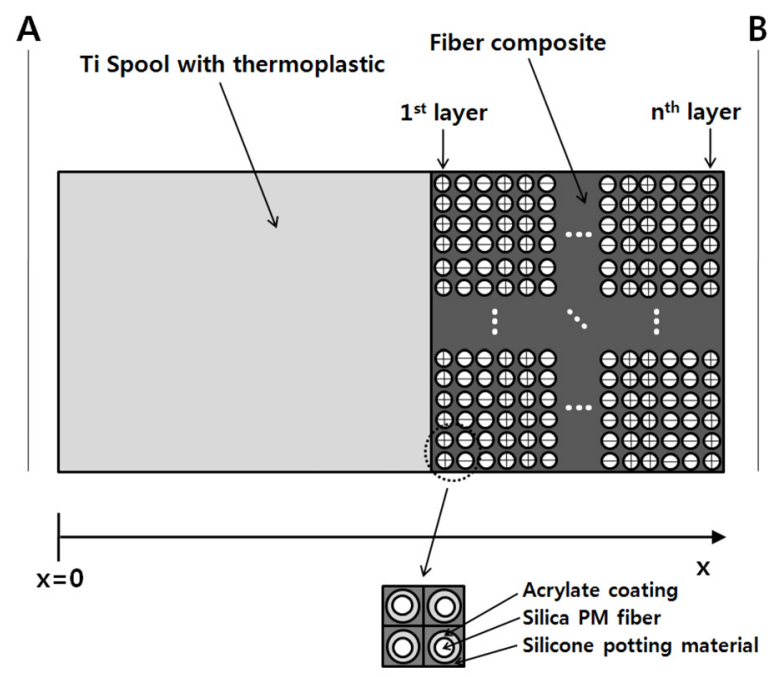

(b)

FIG. 1. Schematic view of (a) fiber coil and (b) its 1-dimensional cross-section with quadrupolar winding pattern (one half coil length is represented by + and the other by -). 
for a fixed coil geometry. There have been two main methodologies to determine the $\Delta \dot{T}$; one is based on the finite element method (FEM) [6], and the other utilizes a numerical calculation with an analytic heat conduction equation [5]. Even though the latter methodology may be less adaptive for correct estimation of $\Delta \dot{T}$, it is very advantageous to take analytic insight into the thermal characteristics in the process of solving the problem. In this study, a one-dimensional analytic heat conduction model based on the following equation is selected to catch the thermal diffusivities of the fiber coil directly [5]:

$$
K \frac{\partial^{2} \Delta T}{\partial x^{2}}=\frac{\partial \Delta T}{\partial t}
$$

where $K$ is the thermal diffusivity of the material. Here, $K$ is a critical constant expressed by thermal-related properties as

$$
K=\frac{k}{\rho c_{p}},
$$

where $k, \rho$, and $c_{p}$ are thermal conductivity, density, and specific heat capacity, respectively. Because the coil is composed of various materials that can be largely divided by two different parts, namely, the metallic spool and arranged fiber encapsulated by potting material, it is necessary to simplify the calculation by treating them as a homogeneous body with a rescaled coordinate, $\bar{x}$ for the $n$th layer:

$$
\bar{x}=\frac{l_{s}}{2 \sqrt{K_{s}}}+\frac{d_{c}}{2 \sqrt{K_{c}}} \cdot n
$$

where $l_{s}$ and $d_{c}$ are the length of metallic spool and the average diameter of fiber composite per layer, and $K_{s}$ and $K_{c}$ are the thermal diffusivity of spool and fiber composite, respectively. For the given initial condition $\Delta T(x, 0)=0$ and the boundary condition $\Delta T(0, t)$, the rescaled coordinate $\Delta \dot{T}(x, t)$ can be calculated numerically. If $\Delta \dot{T}(x, t)$ is determined, the rotation rate error due to the thermal transient perturbation can be also determined by applying the Eq. (1) to following equation [10]:

$$
\Omega_{e}=\frac{\lambda c}{2 \pi L D} \cdot \Delta \phi_{e},
$$

where $\lambda$ and $D$ are the central wavelength of the light source and the average loop diameter of the fiber, respectively.

Note that the transient rate error due to the thermal perturbation is apparently dependent on $d n / d T, K_{s}$, and $K_{c}$ for the given $\Delta T(x, t)$ and fixed coil geometry. This implies that even small changes in these three critical factors can influence the Shupe effect of IFOG. Also, as confirmed in [8], strictly speaking, the Shupe effect is not only a function of the temperature rate but also of temperature itself. As Eq. (1) seems to be solely related to the relative temperature gradient, it is necessary to consider the critical factors as variables on temperature. Therefore, for precise analysis of the Shupe effect, it is indispensable to include the variant effects of $d n / d T, K_{s}$ and $K_{c}$ in terms of temperature change. In this study, temperature dependence on these factors is investigated to identify the variant susceptibility of the Shupe effect on the temperature change.

\section{EXPERIMENTAL PROCEDURE}

The configuration of IFOG tested in this work is depicted in Fig. 2. The light from a broadband erbium-doped fiber source (EDFS) with a $1550 \mathrm{~nm}$ center wavelength is split by a 3-dB optical coupler and then coupled to a fiber which is pigtailed to $\mathrm{LiNbO}_{3}$ integrated optic chip (IOC) which functions as a TM-mode-rejecting polarizer, a Y-branch 3-dB beam splitter, and an electro-optical phase modulator. A polarization maintaining fiber (PMF) with $80 \mu \mathrm{m}$ cladding and $155 \mu \mathrm{m}$ coating diameter, and 1.45 core index is wound in a $40 \mathrm{~mm}$ diameter spool for realization of the fiber coil. The total length of the PMF in the coil is about $900 \mathrm{~m}$, and the fiber coil is composed of 60 fiber layers with a quadrupolar winding pattern. After passing through the fiber coil, two counter propagating light beams are interfered at the common input port of the Sagnac loop, and an interference signal is detected at the photo diode. The rate signal is obtained by a closed-loop approach which is based on the serrodyne modulation and demodulation technique. This ensures a linear and stable scale factor [11].

It is very important to check whether other non-reciprocal error sources in addition to the Shupe effect are present in the measured rate bias of IFOG. The presence of any additional non-reciprocal errors causes overestimation in the analysis because they usually cannot be distinguished from the Shupe effect induced errors. The broad spectrum of the utilized EDFS, about $20 \mathrm{~nm}$, reduces not only the nonreciprocal phase error due to back reflections and backscattering in the Sagnac interferometer [12] but also the Kerr

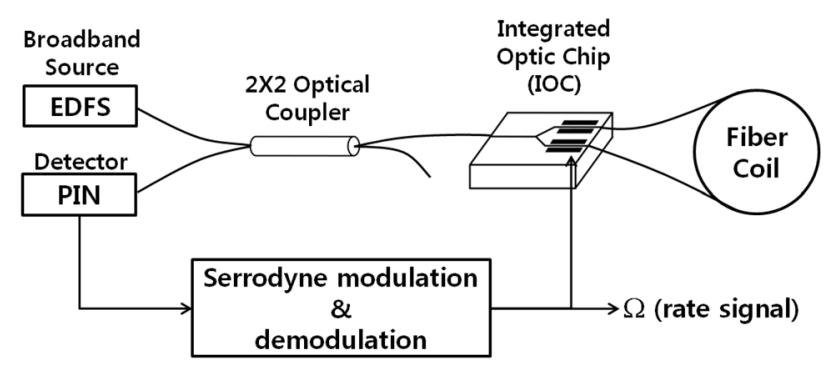

FIG. 2. Schematic view of IFOG configuration. 
effect [13]. The IOC with a high polarization extinction ratio over $60 \mathrm{~dB}$ and birefractive PMF with an h-parameter of less than $10^{-5}$ utilized in the fiber coil can suppress the phase error due to the effect of polarization cross-coupling to a considerably lower level [14-15]. The Faraday bias error which is sensitive to magnetic fields is also eliminated by using a magnetic shield case with highly permeable metal [16]. Before the experiment to analyze the Shupe effect, it was confirmed that this configuration has a very small phase error of less than $10^{-7}$ radian by testing the IFOG in a static environment without thermal transient perturbation. Because the peak level of the expected phase error due to transient thermal perturbation in this study is larger than an order of $10^{-6}$, the configuration can be considered suitable for analyzing the Shupe effect.

After the preliminary investigation, experiments with the Shupe effect were performed at several surrounding temperatures, namely, $-30^{\circ} \mathrm{C}, 25^{\circ} \mathrm{C}$, and $50^{\circ} \mathrm{C}$, to identify temperature dependence on the critical factors, $d n / d T, K_{s}$, and $K_{c}$. The IFOG was mounted in the temperature chamber, and the initial surrounding temperature condition was achieved as a state of thermal equilibrium. Then, an electrically induced local heat source was turned on so that thermal transient perturbation was applied asymmetrically to the fiber coil by exposing one of its sides to thermal flux coming from the heat source. The rate signal of the IFOG and temperature information were measured for 3 hours. The two thermocouples were located at the center of the fiber coil and outside of the fiber coil. In this work, thermal perturbation is required to have adequate temperature variation over a range of several degrees without affecting significant changes in the critical factors while confirming the Shupe effect in the measured results. The relatively long measurement duration of 3 hours is an important condition. The long-term stability of the rate signal including the steadystate are identified for the given thermal perturbation; such necessary information is very helpful in analyzing the effects more accurately.

\section{RESULTS AND DISCUSSION}

For the given coil geometry and $\Delta T(x, t), d n / d T, K_{s}$, and $K_{c}$ were extracted by applying the analytic model described in Section 3 to the measured rate error of the IFOG during the experiment. The measured boundary conditions at the two thermocouples are shown in Fig. 3, and the measured transient rate error due to the perturbation is shown in Fig. 4. The Shupe effect induced rate error could be obtained after eliminating the noise in the measured output by using the 100-second adjacent average and compensating the fixed bias by assuming that there is no Shupe effect induced error in the steady- state. The fitted values in Fig. 4 are analyzed results with the extracted three critical factors, and they seem to be almost the same as the measured results. This implies that the analysis was accurate, and the analytic model proposed in this study can be applied in compensation of the transient rate error. In Fig. 5, the compensated outputs using the fitted results are shown.

Actually, there may be some overestimation in identifying

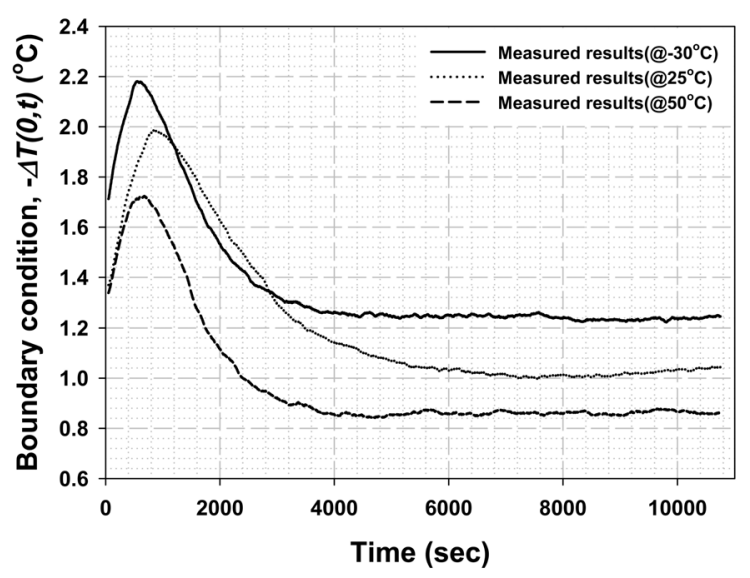

FIG. 3. Measured boundary conditions.

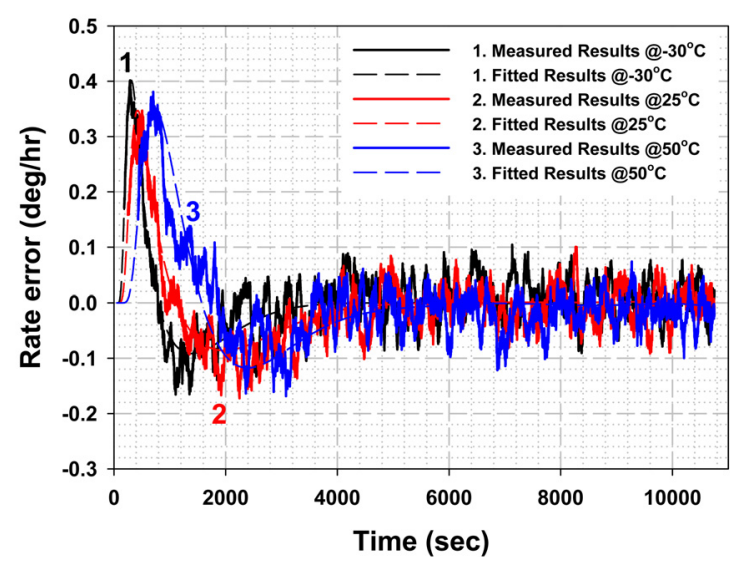

FIG. 4. Comparison of the measured and fitted rate errors due to the Shupe effect with the radial boundary conditions seen in Fig. 3.

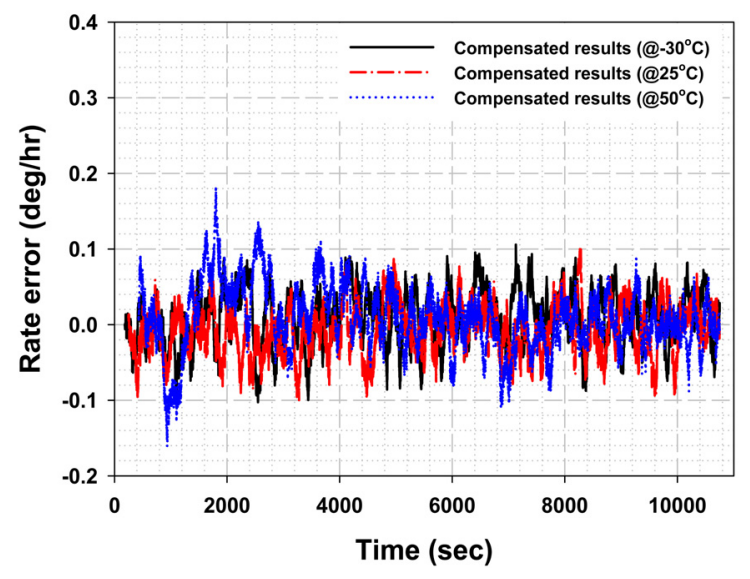

FIG. 5. Compensated output obtained by subtracting the fitted values from the measured results presented in Fig. 4. 
the correct values for the thermal characteristics. This is because the experiments and analytic model in this study are not perfect to characterize correct values for $d n / d T, K_{s}$, and $K_{c}$. For example, non-ideal quadrupolar winding in the fabrication of the fiber coil can cause overestimation in analyzing the $d n / d T$. In addition, the one-dimensional simple analytic model cannot treat various thermal features of the non-uniform radiant heat flux utilized during the experiment which are also important variables determining the Shupe effect. However, despite such imperfections of the experiments and the analytic model, they are still acceptable in that the temperature-induced changes in the Shupe effect can be analyzed effectively with the extracted results.

All the extracted critical factors from each experiment are summarized in Figs. 6 and 7. It is possible to confirm two noteworthy tendencies in Figs. 6 and 7. One is that $K_{s}$ and $K_{c}$ decreased, and another is that $d n / d T$ increased with increase in the surrounding temperature. Considering that all the constituting materials of the fiber coil presented in Fig. 1 (b) have almost linearly decreased features in thermal diffusivity, while increased features in thermal expansion

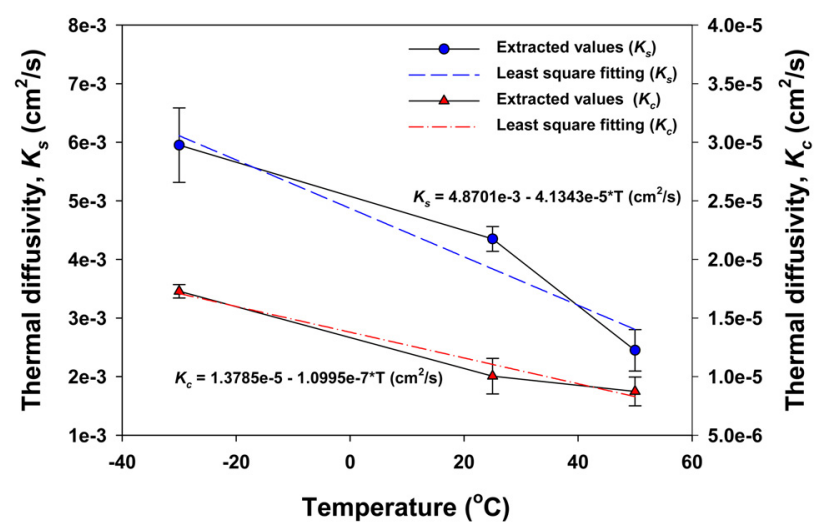

FIG. 6. Extracted thermal diffusivities of spool and fiber composite from the measured results.

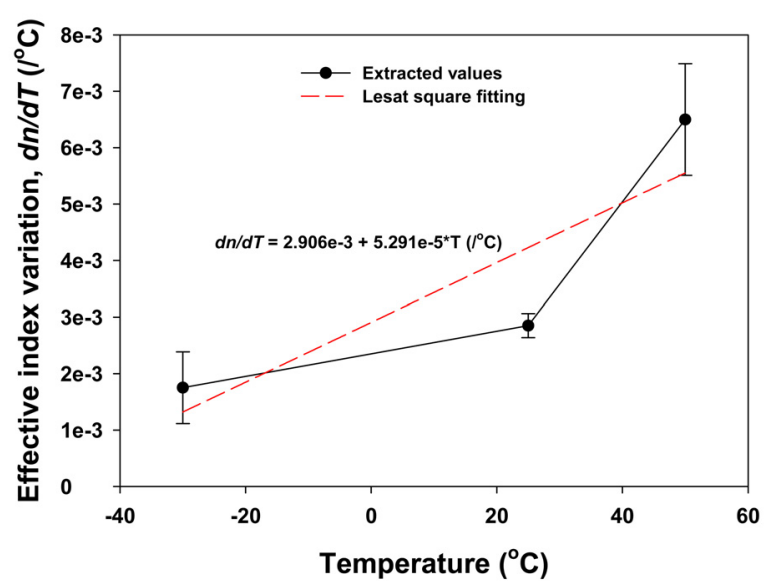

FIG. 7. Extracted temperature induced changes of fiber mode index from the measured results. coefficient which can enhance the stress induced index change as the temperature of the fiber coil is increased [17], these results seem reasonable. Although it is difficult to estimate the correct values for the critical factors by using the thermal characteristics of the constituting material themselves, this fact is sufficient to conclude that the comprehensive thermal characteristics of the fiber coil have the same tendencies as the constituting materials on the temperature changes. The fractional changes in the extracted values can be evaluated effectively by assuming that they comply with linear temperature increase as seen in Figs. 6 and 7. By applying the $1^{\text {st }}$-order least-square fitting technique to the extracted values, the constant change rates of $K_{s}, K_{c}$, and $d n / d T$ on temperature increase were analyzed to be $-41 \mathrm{ppm} \cdot \mathrm{cm}^{2} / \mathrm{s} /{ }^{\circ} \mathrm{C}$, $-0.11 \mathrm{ppm} \cdot \mathrm{cm}^{2} / \mathrm{s} /{ }^{\circ} \mathrm{C}$, and $53 \mathrm{ppm} /{ }^{\circ} \mathrm{C}^{2}$, respectively.

The analyzed simple linear equations from Figs. 6 and 7 are very advantageous when they are utilized in the estimation of the Shupe effect based on the analytic model because they can generate reasonable values on the critical factors at arbitrary temperatures within the considered range. The calculated Shupe effect induced biases for five different temperatures utilizing the extracted linear equations and the analytic model are presented in Fig. 8. The boundary conditions which are necessary for the calculation of the Shupe effect induced biases are assumed to be same as that measured at $25^{\circ} \mathrm{C}$. The calculation results in Fig. 8 show that the peak-to-peak quantity and the zero-crossing time of the Shupe effect induced bias increase with increase in the surrounding temperature. These calculation results have a tendency similar to that of the measured results seen in Fig. 4 in the aspect of the broadening of the transience. However, the variant features of the peak-to-peak quantity seen in Fig. 8 do not seem similar to that of Fig. 4. The peak-to-peak quantity of the Shupe effect induced bias measured at $-30^{\circ} \mathrm{C}$ is obviously larger than that of the results measured at higher temperatures of $25^{\circ} \mathrm{C}$ and $50^{\circ} \mathrm{C}$. These differences between Figs. 4 and 8 are due to the different boundary conditions in the process of estimating

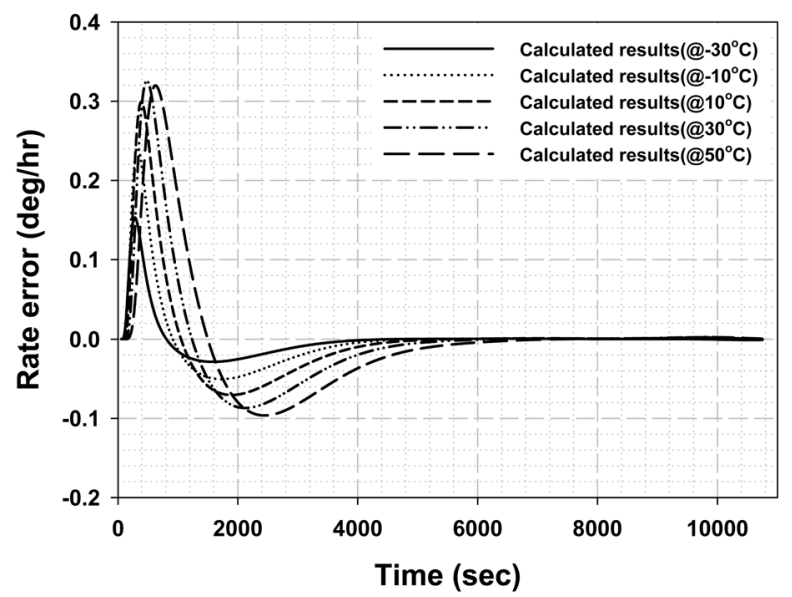

FIG. 8. Calculated rate error with the three linear equations of Figs. 6 and 7, and the measured boundary condition at $25^{\circ} \mathrm{C}$. 


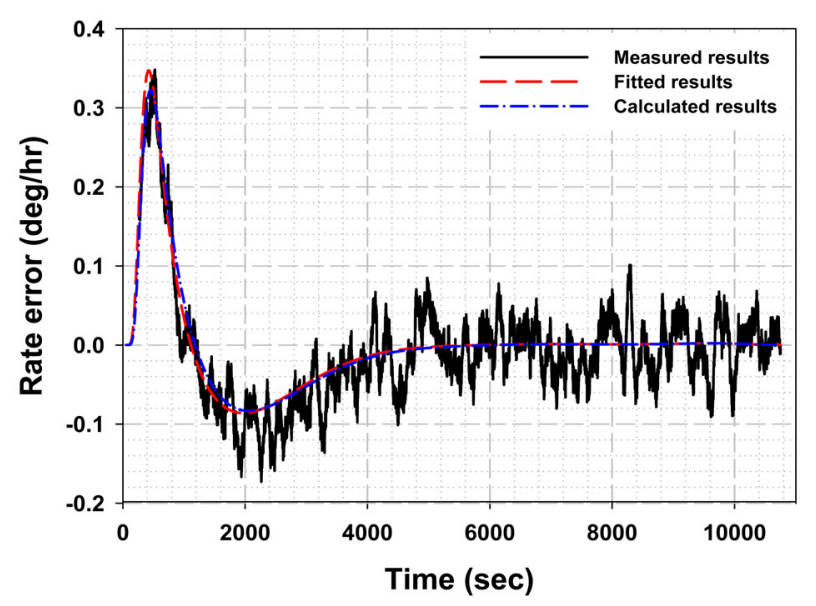

FIG. 9. Comparison of the measured, fitted, and calculated rate error at $25^{\circ} \mathrm{C}$.

the Shupe effect induced error. Considering that the nominal values of the boundary condition measured at $-30^{\circ} \mathrm{C}$ are larger than those of the boundary condition measured at $25^{\circ} \mathrm{C}$ and $50^{\circ} \mathrm{C}$, as seen in Fig. 3, the variant features in the peak-to-peak quantity of the Shupe effect induced bias in Fig. 4 are essentially identical to the features of Fig. 8 .

From the results of Figs. 4 and 8 , it can be easily confirmed that the variation of the Shupe effect induced error per unit of temperature change is very small relative to the intrinsic outputs in steady-state, which drift and swing within the bounded range of $\pm 0.1 \mathrm{deg} / \mathrm{hr}$. Because the Shupe effect is analyzed based on the measured outputs of IFOG, any measurements for numerous surrounding temperature that do not exhibit distinct variations in the Shupe effect induced error more than the intrinsic bias uncertainty of IFOG can be supernumerary. Because the transient-state is usually inclined to have poorer performance than the steady-state section even when the Shupe effect induced error is not considered, for an accurate analysis, it is more important to achieve good bias repeatability in the transient-state. In this study, it was confirmed by taking repeated measurements at the predetermined surrounding temperatures that bias repeatability in the transient-state is as good as that in the steady-state section. The confirmation of bias performance and the corresponding three temperature points for measurement and analysis in this study are the minimum suitable conditions for analyzing the Shupe effect with the extracted variant equations over the considered range of temperatures. For $25^{\circ} \mathrm{C}$, the calculated Shupe effect induced error with the variant equations is presented together with the fitted results based on the analytic model and the measured data in Fig. 9. It can be easily found out that there are negligible differences of less than $0.03 \mathrm{deg} / \mathrm{hr}$ between the fitted and calculated Shupe effect induced bias error.

In Table 1, the results of Shupe effect compensation using the linear equations are summarized. The compensation is carried out with only the transient data up to initial 1
TABLE 1. Shupe effect compensation results using linear equations presented in Fig. 6 and 7

\begin{tabular}{c|c|c|c|c}
\hline \hline \multicolumn{2}{c|}{ Temperature $\left({ }^{\circ} \mathrm{C}\right)$} & -30 & 25 & 50 \\
\hline $\begin{array}{c}\text { Bias } \\
\text { instability, } \\
1 \sigma(\mathrm{deg} / \mathrm{hr})\end{array}$ & $\begin{array}{c}\text { Before } \\
\text { compensation }\end{array}$ & 0.1278 & 0.1086 & 0.1266 \\
\cline { 2 - 5 } & $\begin{array}{c}\text { After } \\
\text { compensation }\end{array}$ & 0.0485 & 0.0354 & 0.0703 \\
\hline
\end{tabular}

hour of the measured results for 3 hours and the presented values in Table 1 are worst cases of the compensated results. Even though the compensation is not as precise as the results with the extracted values seen in Fig 5, this compensation still considerably improves the bias performance of the IFOG over a wide range of temperatures.

\section{CONCLUSION}

Temperature dependence on the Shupe effect of IFOGs was analyzed in terms of the thermal characteristics of the fiber coil. It was confirmed that thermal characteristics, such as diffusivity and temperature-induced change in the fiber mode index of the fiber coil, were critical factors that determined the Shupe effect of IFOGs. By applying an analytic model with 1-dimensional heat conduction and the geometry of the fiber coil to the measured transient responses of an IFOG, the critical factors were accurately identified. Temperature-induced changes in the critical factors were also analyzed by experiments at three different temperatures, and these factors exhibited variant tendencies to temperature changes. They were confirmed to be essential in compensating the transient effect over a wide range of temperatures.

\section{REFERENCES}

1. R. A. Bergh, H. C. Lefervre, and H. J. Shaw, "An overview of fiber-optic gyroscopes,” J. Lightwave Technol. 2, 91-107 (1984).

2. R. Ulrich, "Fiber-optic rotation sensing with low drift," Opt. Lett. 5, 173-175 (1980).

3. D. M. Shupe, "Thermally induced nonreciprocity in the fiber-optic interferometer," Appl. Opt. 19, 654-655 (1980).

4. N. J. Frigo, "Compensation of linear sources of nonreciprocity in Sagnac interferometers," Proc. SPIE 412, 268-271 (1983).

5. C. M. Lofts, M. Parker, and C. C. Sung, "Investigation of the effects of temporal thermal gradients in fiber optic gyroscope sensing coils," Opt. Eng. 34, 2856-2863 (1995).

6. O. F. J. Tirat and J. F. M. Euverte, "Finite element model of thermal transient effect in fiber optic gyro," Proc. SPIE 2837, 230-238 (1996).

7. H. C. Lefervre, The Fiber-optic Gyroscope (Artech House, Inc., Norwood, MA, USA, 1993), Chapter 6.

8. A. Cordova, D. J. Bilinski, S. N. Fersht, G. M. Surabian, 
J. D. Wilde, and P. A. Hinman, "Sensor coil for low bias fiber optic gyroscope," U. S. patent 5371593 (1994).

9. F. Mohr and F. Schadt, "Rigorous treatment of fiberenvironmental interactions in fiber gyroscopes," in Proc. IEEE REGION 8 SIBIRCON 2008 (Novosibirsk Scientific Centre, Russia, July 2008), pp. 372-375.

10. H. C. Lefervre, The Fiber-optic Gyroscope (Artech House, Inc., Norwood, MA, USA, 1993), Chapter 2.

11. J. A. Pavlath, "Closed-loop fiber optic gyros," Proc. SPIE 2837, 46-60 (1996).

12. R. A. Bergh, H. C. Lefevre, and H. J. Shaw, "All-singlemode fiber-optic gyroscope with long-term stability," Opt. Lett. 6, 502-504 (1981).

13. K. Petermann, "Intensity-dependent nonreciprocal phase shift in fiber-optic gyroscopes for light sources with low coherence," Opt. Lett. 7, 623-625 (1982).

14. W. K. Burns and R. P. Moeller, "Polarizer requirements for fiber gyroscopes with high-birefringence fiber and broad-band sources," J. Lightwave Technol. 2, 430-435 (1984).

15. W. S. Choi and M. S. Jo, "Accurate evaluation of polarization characteristics in the integrated optic chip for interferometric fiber optic gyroscope based on path-matched interferometry," J. Opt. Soc. Korea 13, 439-444 (2009).

16. K. Petermann, K. Bohm, and E. Weidel, "Sensitivity of a fiber-optic gyroscope to environmental magnetic fields," Opt. Lett. 7, 180-182 (1982).

17. A. Goldsmith, Handbook of Thermophysical Properties of Solid Materials (Macmillan, New York, USA, 1961). 